\title{
Inteligencia emocional del líder de la empresa turística a partir de una intervención de coaching *
}

\author{
Emotional Intelligence of Leaders at a Tourism Company After a Coaching \\ Intervention
}

\author{
César Omar Velázquez-Vega \\ Doctorando en Desarrollo Sostenible, Universidad de Quintana Roo, \\ Cozumel - México, ceveve@gmail.com \\ Alfonso González-Damián \\ Doctor en Ciencias Sociales, Universidad de Quintana Roo, \\ Cozumel - México, gonzalezd@uqroo.edu.mx
}

Cómo citar / How to cite

Velázquez-Vega, C. O., González-Damián, A. (2021). Inteligencia emocional del líder de la empresa turística a partir de una intervención de coaching. Revista CEA, v. 7, n. 15, e1842.

https://doi.org/10.22430/24223182.1842

Recibido: 24 de febrero de 2021

Aceptado: 15 de junio de 2021

\section{Resumen}

El desarrollo de la inteligencia emocional es transcendental para enfrentar los objetivos organizacionales de la empresa turística. El propósito del estudio fue determinar la existencia de una influencia significativa en la inteligencia emocional de 56 líderes de una empresa turística después de una intervención de coaching enfocada en estas habilidades. Se utilizó la herramienta de Reuven Baron para medirla antes y después de la intervención de coaching. Las variables componentes fueron evaluadas mediante un modelo de ecuaciones estructurales por mínimos cuadrados parciales aplicado a los resultados de cada momento de evaluación y fueron comparados mediante pruebas T para muestras pareadas. Los resultados no arrojaron diferencias estadísticamente significativas de mejora en la inteligencia emocional de los líderes tras la intervención de coaching. Esto abrió la discusión respecto a estructuras de intervención más adecuadas para impactar positivamente en la inteligencia emocional de los líderes de la empresa turística. Se concluye que no resalta una mejora significativa en aplicaciones grupales de doce horas, por lo que se hace necesario afinar las características del coaching.

\footnotetext{
* Este artículo se deriva del proyecto titulado «Inteligencia emocional del líder de la empresa turística a partir de una intervención de coaching» y ha sido financiado con recursos del Consejo Nacional de Ciencia y Tecnología (CONACYT) de México.
} 
Palabras clave: liderazgo organizacional, comportamiento empresarial, coaching, inteligencia emocional, empresa turística.

Clasificación JEL: M12, M53.

\title{
Highlights
}

- No se soporta la influencia de aplicaciones coaching sobre el desarrollo de la inteligencia emocional en un grupo de líderes del sector turístico en México.

- Para que las intervenciones de coaching sean efectivas, éstas deberían ser integrales y de más de doce horas de duración.

- Se evidencia la necesidad de que la empresa turística atienda el manejo emocional de sus líderes y colaboradores.

\begin{abstract}
Developing emotional intelligence is essential to face the organizational objectives of tourism companies. This study aimed to determine if there was a significant influence of a coaching intervention on the emotional intelligence of 56 leaders at a tourism company. The Reuven Bar-On model was used to measure their emotional intelligence before and after the coaching intervention. The component variables were evaluated using partial least squares structural equation modeling applied to the results at each evaluation moment and compared using t-tests for paired samples. The results did not reveal statistically significant differences in terms of improvement of the leaders' emotional intelligence after the coaching intervention. This opens the discussion about the most adequate intervention structures to have a positive impact on the emotional intelligence of tourism companies' leaders. It was concluded that the 12-hour group intervention did not produce a significant improvement; hence, the characteristics of the coaching should be adjusted.
\end{abstract}

Keywords: Organizational leadership, corporate behavior, coaching, emotional intelligence, tourism company.

JEL classification: M12, M53.

\section{Highlights}

- Corporate leaders' emotional skills have an impact on organizational performance.

- Effective coaching interventions should be comprehensive and last more than twelve hours.

- Tourism companies should address the emotion management of their leaders and employees.

- Leadership is strongly influenced by emotional intelligence.

\section{INTRODUCCIÓN}

Existen estudios, de acuerdo con Petrides et al. (2016), que han puntualizado la relevancia de las habilidades emocionales integradas en la inteligencia emocional (IE) y su impacto en el desempeño exitoso del individuo (Mestre Navas et al., 2017), pero ninguno enfocado en el líder de la empresa turística de la Riviera Maya. Esto atrae la atención de esta investigación, pues las habilidades emocionales se convierten en una ventaja natural cuando se desarrollan funcional y eficazmente 
(Diez Farhat y Sánchez, 2017) debido a que estas pueden potencializar el rendimiento, eficacia y compromiso del líder en su desempeño profesional (Lopez-Zafra et al., 2017).

Es el desarrollo de las habilidades integradas en la IE lo que permite contar con más y mejores herramientas para dirigir y apoyar a los colaboradores para superar los desafíos cotidianos, encaminándolos al logro de objetivos (Parr et al., 2016). Para el ejercicio del liderazgo, la IE juega un rol primordial y determinante (Lee y Chelladurai, 2017), pues un líder efectivo organiza las actividades necesarias y asegura que se hagan, es humano en su trato y mantiene relaciones eficaces con su equipo de trabajo (Fernández-Abascal, 2009), elementos visibles cuando se cuenta con niveles de IE superiores.

Quien busca involucrarse en un estudio relacionado con la empresa turística se enfrenta a la necesidad de determinar un marco teórico conveniente (González-Damián, 2017) y los desarrollos teóricos en este campo están muy relacionados con una orientación teórica en la sociología contemporánea (Cohen y Cohen, 2012), desde donde se busca identificar los patrones sociales y su pertinencia. Un camino acotado del estudio de la sociología procura diseñar un sólido campo teórico que facilite una aproximación sistemática al cuerpo emocional de la sociedad (Ariza, 2016); es ahí donde descansa esta investigación, resaltando la necesidad de estudiar la IE de la fuerza de trabajo turística como parte de una sociedad anfitriona que es responsable de que la experiencia turística se logre (González-Damian, 2018; Bermúdez-Tirado et al., 2016), destacando que es una industria que está experimentando diversas transformaciones y requiere que el liderazgo sea ágil, flexible y guíe a sus seguidores a través de estos cambios (Kim y Jeong, 2018).

Las emociones son la base del pensamiento racional (Acosta Palomeque et al., 2017; Chang, 2017) y gobiernan de forma silenciosa la manera en que el individuo interactúa con su entorno (Fernández Moya, 2016), se desarrolla en él y se comunica consigo mismo. La autorregulación emocional suele ser auto informada (Grandey y Gabriel, 2015) y desde el lenguaje emocional se transforma la comunicación propia para gestionar y controlar el propio yo (Prieto Egido, 2017) y los comportamientos en consecuencia, lo que destaca la relevancia de que el líder de la empresa turística cuente con habilidades emocionales desarrolladas que favorezcan el éxito en su gestión.

La literatura afirma que la participación en intervención de coaching puede impactar en las estrategias de autorregulación emocional del individuo (Corti y Gelati, 2020) y, aplicado al sector turístico, tiene el objetivo de optimizar los recursos y la satisfacción de los trabajadores creando motivación y compromiso (Montolio, 2014). Su aplicación en el campo empresarial se considera entre las cinco principales para el efectivo desarrollo del liderazgo (Baquero Barato y Rodríguez-Moneo, 2016), por lo que se rescata e incluye en esta investigación. Se utiliza como un proceso de mejora del individuo en diferentes ambientes y se despliega en una amplia gama de prácticas (Stelter, 2014), desde donde es posible generar conciencia para clarificar objetivos y estrategias (Sandoval Lentisco y López Martínez, 2017).

Con base en estos antecedentes se planteó el propósito de determinar si existe un efecto positivo y significativo en la IE de un grupo de líderes, mediante una intervención de coaching diseñada exprofeso para tal finalidad. Dada la naturaleza operativa de las empresas turísticas en la Riviera Maya en México, los líderes participaron en la intervención, sin dejar a un lado la operación normal de la empresa y realizando sus actividades cotidianas en los puestos que cada uno desempeña en la misma, 
lo cual, en sí mismo, constituyó tanto una limitación como una forma de realizar un experimento natural (Nass Kunstmann y Merino, 2008). El texto presenta a continuación una revisión de literatura sobre el líder, la inteligencia emocional, el coaching y se aterriza en el estudio de las empresas turísticas. Se presenta la metodología del estudio, los resultados, la discusión y las conclusiones.

\section{MARCO TEÓRICO}

Hollander (1980) expuso que el líder con habilidades emocionales superiores lograba relacionarse con respeto y afecto. Desde este postulado se puede aseverar que un alto nivel de IE logra predecir la efectividad al ejercer el liderazgo (Kotzé y Venter, 2011).

Edú Valsania et al. (2016) mostraron que el líder puede influir en el éxito de sus colaboradores y motivarlos al logro de objetivos superiores a los esperados. El ejercicio del liderazgo no es un contexto individual, por el contrario, el líder forma parte de un equipo y a su vez de una organización, por lo que el impacto de su desempeño y resultados es amplio. Aunque no exista un perfil de líder definido, pues este se conforma por medio de diferentes personalidades (Parr et al., 2016), invariablemente su IE determina su desempeño y por medio de ella influye en sus equipos de trabajo y en sus resultados (Lopez-Zafra et al., 2017).

En el contexto actual, en las economías globalizadas de este siglo, el líder se convierte en un activo valioso y significativo para las empresas cuando es capaz de enfrentarse a las tareas y a los desafíos diarios del entorno comercial de modo eficaz y efectivo (Salomaa y Mäkelä, 2017). Estas mismas economías forman parte de un mundo en constante movimiento, expuesto a competencias voraces del entorno empresarial, por lo que es transcendental conocer la relevancia de la IE del líder para vislumbrar y proyectar los éxitos de las organizaciones (Diez Farhat y Sánchez, 2017), pues elevados niveles de ella favorecen la construcción de pensamientos positivos y logra relaciones laborales satisfactorias (Băeşu y Bejinaru, 2015).

Para poder generar un crecimiento organizacional se requiere cultivar la IE de los empleados, pues solo así lograrán atravesar la inminente resistencia al cambio (Gelaidan et al., 2016; Issah, 2018). De hecho, el líder tiene un rol tan relevante, que puede determinar el éxito o el fracaso de una organización de acuerdo con su visión y desempeño (Semuel et al., 2017), pues su efectividad se mide por la capacidad de influir positivamente en sus subordinados, impactando a la organización en tanto a sus logros y éxitos (Madanchian et al., 2017; Bonesso et al., 2015).

Las organizaciones modernas requieren colaboradores con la capacidad de generar intervenciones positivamente emocionales en sus lugares de trabajo (Vallejo Altamirano et al., 2017), pues los comportamientos negativos tienen más consecuencias que los positivos y pueden ser más difíciles de cambiar (Larsson et al., 2017). Donde hay una correcta gestión se reducen costos mejorando los beneficios en la cadena de valor (Ibarra Michel, 2014), aportando valía a la sostenibilidad de la empresa. Esta gestión demanda fomentar en el líder y en sus equipos la comprensión y entendimiento de la IE (Priyanka y Taranjeet, 2016).

\section{La inteligencia emocional}

Reuven Bar On (2006) comienza con su investigación sobre la IE en la década de los ochenta, y la define como esas habilidades sociales y emocionales que influyen en el éxito del individuo, logrando 
un modelo de quince constructos que convergen en uno solo, denominado cociente emocional. Entre estos constructos se incluyen habilidades para estar consciente de uno mismo, para comprender las fortalezas y debilidades propias y poder expresar el pensamiento y los sentimientos de una manera constructiva desde factores intrapersonales e interpersonales. Considera de igual manera el manejo del estrés, la adaptabilidad y la visión optimista y alegre de la vida.

La IE es ahora un tema relevante para la investigación, así como los semblantes emocionales y sociales dentro de este concepto (Bar-on, 2013). La herramienta de Bar-On, que probablemente sea la mejor para evaluar la IE (Bisquerra Alzina et al., 2006) es la elegida en este trabajo, pues es la más acertada al considerar habilidades intra e interpersonales del individuo (Bar-On, 2006). Este tipo de modelo, llamado mixto, considera destrezas y rasgos (Fragoso-Luzuriaga, 2015).

Cuando el individuo carece de la capacidad para identificar sus verdaderos sentimientos se encuentra expuesto ante estos; por el contrario, cuando logra identificarlos, consigue manejarlos y obtener lo mejor de sí mismo en su desempeño diario (Jacka, 2018). A la IE se le involucra con los efectos drásticos en el rendimiento y éxito de una organización, y en los colaboradores tiene una gran importancia para codificar actitudes y comportamientos (Ahmad et al., 2017). Para Goleman (1995) la IE se refiere a todo eso que queda afuera de la medición del coeficiente intelectual y que tiene estrecha relación con el éxito. Con todo esto, se clarifica la relevancia constructiva de la IE en el éxito personal y profesional del individuo (Ghoudani et al., 2017).

En un estudio realizado por Seymour (2017), se presentan resultados estadísticos que indican la afectación significativa en las competencias de la IE después de una intervención de coaching a 30 líderes, usando como herramienta la escala de Reuven Bar-On. La relevancia de este modelo se consolida por la necesidad de liderazgo efectivo en las organizaciones, que está respaldada por diversos estudios (Kotzé y Venter, 2011).

\section{Liderazgo e inteligencia emocional}

En el mundo actual, las compañías necesitan de la ventaja competitiva que otorga el líder con habilidades que dirigen al éxito, pero esto no se logra solo con la experiencia profesional habitual, pues requiere saber reconocer y manejar sus emociones ya que estas juegan un rol destacable que mejora su efectividad y posibilidades (Özdemir y Özkul, 2016). La evidencia sostiene que el líder con una personalidad proactiva motiva al equipo al logro de objetivos (Lam et al., 2018).

El líder de la empresa turística debe contar con la información certera de las áreas en que se quiere mejorar (Drucker, 2007) y, a su vez, el mismo líder requiere de habilidades que le permitan manejar esta información y su actuar de manera emocionalmente asertiva y efectiva. Dentro del proceso empresarial, el líder trabaja a lado del colaborador, codo a codo, ayudándolo y alentándolo; además de dirigirlo de forma optimista y decidida (Taylor, 1968), solo desde esta plataforma se logra construir los cimientos para una gestión fuerte y efectiva, la cual es una necesidad para que la empresa turística sea sustentable y competitiva (Garcia Reinoso et al., 2020).

El liderazgo está vigorosamente afectado por las habilidades contenidas en la IE, pues son ellos quienes desempeñan el rol de arquitectos de la cultura organizacional y el clima que se respira al interior de ella, esto emana de las emociones que proyecta y provoca en sus colaboradores. Si el líder 
es percibido como injusto por su equipo genera emociones negativas y resultados adversos, además de que instituye una dinámica negativa, la cual la alta dirección de la empresa detecta, esto provoca que se pierda la confianza en él (Ashkanasy y Humphrey, 2014).

El líder ejerce influencias importantes sobre los estados de ánimo y los sentimientos de sus seguidores y compañeros de equipo, principalmente por el efecto del contagio emocional (Ashkanasy y Humphrey, 2011). Conviene resaltar que este contagio germina, aunque el líder, sus seguidores y la empresa en general desconozcan su existencia, importancia o impacto.

De igual forma, el Líder debe considerar en su totalidad el ambiente de acción y lograr definir cuáles son las mejores conductas para aumentar la probabilidad de éxito (Cosans y Reina, 2017), debiendo comprender que su personalidad «jala» a la organización y a su conjunto para bien o para mal (Bauman, 2018).

La noción del líder emocionalmente inteligente capta la atención de la academia, ya que el cuerpo científico ha postulado la relevancia de la IE en el ambiente de trabajo (Mfikwe y Pelser, 2017). EI interés en el tema es tal que se delibera sobre esta inteligencia de la mano del líder y sus roles para lograr la excelencia de la organización (Maqbool et al., 2017). El líder exitoso está obligado a comprender las emociones propias y de su equipo desarrollando la empatía (Özdemir y Özkul, 2016; Boyatzis et al., 2017), concibiendo la relevancia de la IE para ganar la lucha que implica el cambio constante (Băeşu y Bejinaru, 2015). El líder inteligente con sus sentimientos y emociones conduce a las de los demás en una dirección correcta y efectiva (Mathew y Gupta, 2015), siendo así, capaz de provocar el compromiso laboral de los trabajadores y buenas relaciones entre ellos (Ochalski, 2016). Cuando el líder cuenta con un nivel elevado de autoconciencia emocional, consigue de la misma forma el entendimiento de sus creencias y valores, incluyendo sus verdaderos pensamientos y sentimientos en su naturaleza. Este líder debe enmarcar honestidad y responsabilidad, pues se desenvuelve desde una transparencia natural y logra alejarse con facilidad de la vanidad y del interés por conseguir recompensas puramente personales. Aunque este líder se desempeñe con valores y convicciones aprendidas de alguien más, los adapta y personaliza sin perseguir popularidad (Herrero Garcí et al., 2019).

La IE se postula como la que más preeminencia tiene entre todas las habilidades que requiere el líder (Preston et al., 2015), pues influye positivamente en el compromiso de los equipos hacia la organización, y favorece la gestión de las relaciones interpersonales y la conciencia social (Priyanka y Taranjeet, 2016). Desde estos postulados teóricos se resalta la necesidad de una nueva evaluación radical de la formación del líder desde una forma responsable y consiente (Hibbert et al., 2017) y de trabajos que revelen la influencia de la IE en su desempeño, resultados y logro de objetivos.

La IE, a su vez, es definida como la capacidad mental relacionada con las experiencias emocionales (Mayer et al., 2016) y su importancia en la actualidad es premisa de discusión (Ghoudani et al., 2017). De hecho, existe evidencia que refiere que las habilidades incluidas en la IE profetizan una relación con la salud mental, y que a través de una intervención enfocada se puede potencializar (Davis y Humphrey, 2012), lo que en un grupo de líderes de la empresa turística podría representar una importante ventaja competitiva. 


\section{El coaching en inteligencia emocional}

Para Ravier (2009), el coaching es un proceso sustentado en el diálogo entre dos partes, una de ellas genera reflexiones que provocan acción para el logro de metas definidas, la otra conduce el proceso. Para el éxito de este sumario es necesario determinar los objetivos, los medios y requerimientos con la elaboración de un plan de acción; es decir que se define como un proceso dialógico donde las partes concretan una relación como coach (experto) y coachee (cliente) (Sandoval Lentisco y López Martínez, 2017).

El coaching parece contar con un campo de acción más amplio que la psicoterapia y el asesoramiento, ya que extrae las más diversas disciplinas y profesiones (Abravanel y Gavin, 2017). El coaching ejecutivo funciona como una intervención para el desarrollo del líder, implicando el dominio de nuevas habilidades (Abravanel y Gavin, 2017); se refiere a una intervención para el desarrollo del potencial humano, pero es un proceso complejo que requiere cuidado y ética (Pliopas, 2017). Cuando es posible crear una relación confiable y confidencial, se facilita el aprendizaje y mejora del rendimiento del coachee, provocando un estado emocional positivo (Ladyshewsky, 2017). Es un proceso que explora posibilidades para guiar al coachee a lograr metas y mejorar su desempeño (Smith, 2017).

Para The Concise Oxford Dictionary Of Current English (Sykes, 1990), «coaching» es «tutelar, adiestrar, dar indicaciones, comunicar hechos». Este funciona como un acelerador o herramienta educativa que lleva al líder a integrarse en un camino más rápido hacia el logro de sus objetivos (Gregory y Wiles, 2018), además de que funciona como una técnica muy efectiva para desarrollar habilidades por su capacidad de modificar comportamientos de una manera enfocada. Igualmente es la técnica con la más amplia aceptación entre los ejecutivos (Rekalde et al., 2017). El coach realiza un ejercicio consciente de la cultura organizacional y el potencial del coachee para lograr un progreso y de la efectiva comprensión del proceso depende que los resultados trasciendan los niveles contextuales (Athanasopoulou y Dopson, 2018).

Hace más de 20 años que se aplicó por primera vez el término coaching al contexto empresarial, ahora se sabe que esta técnica es la mejor oportunidad para que las empresas afronten los cambios y logren una cultura orientada a las personas que la conforman (Whitmore, 2011). El coaching, como proceso, busca acompañar al coachee a generar cambios significativos para lograr resultados extraordinarios (Muñoz Maya y Díaz Villamizar, 2014).

La herramienta principal del coaching, llamada mayéutica socrática, es una de las líneas de mayor impacto para la educación (Noguera, 2017) y ha trascendido en el tiempo gracias a que logra integrar elementos como la interrogación, la reflexión y el diálogo, los cuales buscan promover el desarrollo analítico del pensamiento. El coaching se conduce como una correspondencia en colaboración, donde el coach no dirige y el coachee define sus propios propósitos para su desarrollo (Salomaa y Mäkelä, 2017).

En palabras del denominado padre del coaching, John Whitmore (2011), en cierto modo todos utilizamos y armonizamos las habilidades emocionales, algunas personas más efectivamente que otras. La evidencia apunta que el coaching, en comparación con otros tratamientos, resulta ser más efectivo, incluso para la prevención de la procrastinación, a diferencia de la capacitación tradicional 
que su efectividad se refleja a sólo establecer una meta (Losch et al., 2016). Para mantener viva la visión y proyección del éxito en la empresa es necesario trabajar con procesos de coaching en los líderes (Muñoz Maya y Díaz Villamizar, 2014).

La teoría justifica que los tratamientos de coaching con modelos integrales, específicos y holísticos demuestran ser efectivas para transformar los comportamientos del líder de manera permanente (Passmore, 2010), por lo que la empresa turística puede considerar estas intervenciones para el líder como procesos seguros de mejora para el desarrollo del potencial humano.

\section{La empresa turística}

La empresa turística es como toda empresa, un sistema que interactúa con su contexto y es influida por él, por lo tanto, resulta impactada por las actitudes y comportamientos de sus integrantes (Koontz, 2000). Esta a su vez forma parte de una industria global que ha sido estudiada con diversas bases científicas, entre las que se encuentran las económico-administrativas (Jafari, 2005). En un sentido muy amplio, la empresa turística es aquella que de manera directa o indirecta se encuentra vinculada con la industria global de los viajes, desde las directamente vinculadas con los viajes, como las empresas de transporte de pasajeros, o las que gestionan su infraestructura, como las de aeropuertos, puertos y carreteras, hasta los que proveen servicios enfocados directamente a los viajeros, como hoteles y restaurantes, hasta las que les brindan servicios en sitios turísticos, aun cuando no fueran enfocados específicamente a ellos, como comercios locales, bancos, estaciones de servicio, entre otras tantas (Sancho et al., 2008) y también de manera acumulada, todas las empresas que forman parte de la cadena de valor de los bienes y servicios que se ofrecen a los viajeros.

Desde una postura organizacional estructural, la empresa turística puede tener fines de lucro, por lo que busca rentabilidad y mantenerse vigente en el mercado. Es una figura que tiene propósitos económicos, y al mismo tiempo, se centra en la sociedad. Busca satisfacer las necesidades de turismo y ocio de las sociedades locales y extranjeras, cuenta con recursos humanos, financieros y materiales, y además depende de las capacidades de servicio de sus miembros para su éxito (Ross, 2006).

En su funcionamiento como organización, la empresa turística requiere generar clientes y a su vez competir en el mercado, por lo que resulta muy importante la figura del líder que efectúe funciones de gerencia (Fayol, 1949; Koontz et al., 2012) para planear, organizar, dirigir, coordinar y controlar. En su accionar, el líder se involucra con un conjunto de vínculos sociales afines entre sí, conformando un entramado de relaciones sociales interpersonales, las que a su vez configuran una red de emociones de carácter recíproco. En este sentido, el líder en la empresa turística juega un papel central, tanto desde el punto de vista de la funcionalidad de la organización, como de la configuración del entramado socioemocional del colectivo intraorganizacional.

Esta situación se ve magnificada en el ámbito de la empresa turística, puesto que, por su carácter enfocado a los servicios de viajes y hospitalidad, las relaciones interpersonales incorporan, no únicamente las establecidas entre los miembros de la misma organización, sino una de manera permanente con los usuarios de los servicios: los turistas. Es en este sentido que a la empresa turística le resulta necesario considerar la comprensión y desarrollo de la IE de sus empleados (Koc, 2019). 


\section{METODOLOGÍA}

Existen numerosos modelos y medidas de IE, sin embargo, para el propósito del estudio se considera la medida más adecuada la escala de Reuven Bar-On. Los 15 componentes de este modelo representan un tipo jerárquico de dispositivos que convergen en un único, conocido como cociente emocional (CE). El modelo Bar-On proporciona la base teórica para este coeficiente (Dawda y Hart, 2000), el cual es una muestra representativa de habilidades emocionales y sociales interrelacionadas, que determinan qué tan efectivamente entendemos y expresamos emociones, comprendemos a los demás y nos relacionamos con ellos enfrentando las demandas diarias. De acuerdo con este modelo, ser emocional y socialmente inteligente es comprenderse y expresarse con eficacia, comprender y relacionarse bien con los demás y enfrentar con éxito las demandas, desafíos y presiones diarias (BarOn, 2006).

Los resultados obtenidos de las aplicaciones de la herramienta de Reuven Bar-on antes y después de la intervención de coaching se procesaron mediante un análisis estadístico de ecuaciones estructurales en el programa libre RStudio con base en el modelo de Sánchez (2013) PLSPM, durante el último bimestre del 2018.

La intervención de coaching se realizó a una población de 56 personas, 43 hombres y 13 mujeres, todos líderes en la misma empresa turística del ramo hotelero cinco estrellas en la Riviera Maya, la cual tuvo una duración de doce horas, divididas en dos días. En los análisis se descartaron dos sujetos por no haber realizado el cuestionario final.

La intervención se diseñó con base en el modelo de coaching holístico de Seymour et al. (2017), considerando las habilidades de la IE del modelo de Reuven Bar-on intituladas: Autoconcepto, Autorrealización, Autoconciencia emocional, Expresión emocional, Asertividad, Independencia, Relaciones interpersonales, Empatía, Responsabilidad social, Resolución de problemas, Evaluación de la realidad, Control de impuestos, Flexibilidad, Tolerancia al estrés, y Optimismo (Bar-On, 2006) de acuerdo con el modelo teórico postulado por Ugarriza (2001) y construido en consecuencia.

Debido a que el interés en el estudio de estas variables ha crecido exponencialmente, pues se presume que las habilidades emocionales se convierten en una ventaja competitiva cuando el líder las desarrolla, se realizó este trabajo buscando que el resultado sea valioso para la empresa turística de la Riviera Maya al mostrar la preeminencia del coaching para el desarrollo de las habilidades emocionales y la herramienta de Reuven Bar-on para lograr mediciones certeras de la intervención.

Cada participante completó el cuestionario de autoinforme de IE al iniciar y al terminar la intervención de coaching; esta consta de 133 ítems anclados a una escala de Likert de 5 puntos. La confiabilidad y validez de la herramienta se considera aceptable y constante (Bar-On, 2006), además que ha sido arbitrada en varias ocasiones (Carlson et al., 2014; DiPerna y Sandilos, 2014).

Para realizar la comparación de la variación en los resultados, antes y después de la intervención se ajustaron dos modelos de ecuaciones estructurales mediante el enfoque de Partial Least Squares (PLS) de acuerdo con los postulados de Sánchez (2013), y las medias de los resultados estandarizados (scores) de los modelos ajustados fueron comparados mediante prueba T para muestras pareadas. En ambos casos se utilizó el software estadístico R Core Team (2021). 


\section{RESULTADOS}

En la Tabla 1 se muestra el perfil sociodemográfico de los encuestados con la intención de destacar la diversidad de los participantes y esbozar las características más relevantes. En esta información resalta que el departamento donde los líderes ejecutan sus funciones es, principalmente, en supervisión, coincidiendo en el ingreso percibido como mayoría entre 15 y 20 mil pesos mexicanos; esa mayoría, según los datos presentados, oscila en los 37 años. No obstante, prevalece una escasez de estudios que permitan relacionar la edad adulta con los niveles de IE (Bernarás et al., 2011).

Tabla 1. Características sociodemográficas de los participantes Table 1. Sociodemographic characteristics of the participants

\begin{tabular}{|c|c|c|}
\hline $\mathrm{N}$ & 5 & \\
\hline \multirow{2}{*}{ Género } & Mujeres & .232 \\
\hline & Hombres & .768 \\
\hline \multirow{5}{*}{ Departamento } & Gerencia & .092 \\
\hline & Supervisión & .574 \\
\hline & Coordinador & .074 \\
\hline & Jefatura & .166 \\
\hline & Administrativo & .092 \\
\hline \multirow{5}{*}{ Sueldo en peso mexicano } & $\$ 10000$ a $\$ 14999$ & .127 \\
\hline & $\$ 15000$ a $\$ 20999$ & .490 \\
\hline & $\$ 21000$ a $\$ 25999$ & .200 \\
\hline & $\$ 26000$ a $\$ 30999$ & .054 \\
\hline & $\$ 31000+$ & .127 \\
\hline \multirow{7}{*}{ Edad } & Min & 26 \\
\hline & 1st Qu & 32.75 \\
\hline & Median & 38 \\
\hline & Mean & 37.55 \\
\hline & 3rd Qu & 42.25 \\
\hline & Max & 51 \\
\hline & $S d$ & 6.54 \\
\hline
\end{tabular}

Fuente: elaboración propia.

En la Tabla 2 se presentan los resultados del análisis de fiabilidad y unidimensionalidad realizado a la escala en su aplicación al grupo antes del coaching mediante ecuaciones estructurales por mínimos cuadrados parciales (PLS). Con base en el criterio generalmente aceptado para los valores umbrales de Alpha (Cronbach Alpha) y rho (Dillon-Goldstein rho) de 0.7, se observa que 8 de los 15 constructos para el caso de Alpha y todos los constructos para el caso de rho reflejan valores de fiabilidad compuesta y unidimensionalidad aceptables. Por su parte, como puede verse en las columnas AVE (Varianza Promedio Extraída) y comunalidad, se observa que 14 de los 15 constructos reflejan valores aceptables, por arriba del umbral de 0.5 . En conjunto los valores indican una aceptable validez para los valores que arroja la escala en cada uno de los constructos medidos para la muestra obtenida. 
Tabla 2. Resultados de fiabilidad y unidimensionalidad de la escala

Table 2. Reliability and unidimensionality results of the scale

\begin{tabular}{lcccc}
\hline & $\alpha$ & Rho & AVE & Comunalidad \\
\hline Autoconcepto & .834 & .883 & .604 & .604 \\
\hline Autorrealización & .631 & .802 & .573 & .573 \\
\hline Autoconciencia emocional & .705 & .836 & .625 & .625 \\
\hline Expresión emocional & .813 & .877 & .639 & .639 \\
\hline Asertividad & .730 & .847 & .650 & .650 \\
\hline Independencia & .677 & .823 & .605 & .605 \\
\hline Relaciones interpersonales & .679 & .806 & .513 & .513 \\
\hline Empatía & .801 & .863 & .557 & .557 \\
\hline Responsabilidad social & .710 & .822 & .538 & .538 \\
\hline Resolución de problemas & .678 & .805 & .508 & .508 \\
\hline Prueba de la realidad & .841 & .883 & .557 & .557 \\
\hline Control de impulsos & .560 & .773 & .527 & .527 \\
\hline Flexibilidad & .560 & .773 & .527 & .527 \\
\hline Tolerancia al estrés & .636 & .786 & .476 & .476 \\
\hline Optimismo & .788 & .855 & .542 & .542 \\
\hline
\end{tabular}

Fuente: elaboración propia a partir de (Bar-On, 2006; Ugarriza, 2001).

Una vez validada la escala, se realizó un segundo análisis mediante PLS a los datos después de la intervención de coaching, con lo que se obtuvieron dos modelos ajustados, cada uno a distintos momentos en el mismo grupo, antes y después.

De los modelos ajustados se tomaron los «scores», o valores estandarizados de la IE total y de cada uno de sus constructos constitutivos. A estos scores se les aplicó una prueba T de comparación de medias para grupos pareados entre ambos conjuntos de datos. Los resultados del comparativo por constructo, así como sus grados de libertad, se presentan en la Tabla 3.

Tabla 3. Resultados comparativos por constructo y grados de libertad

Table 3. Comparative results by construct and degrees of freedom

\begin{tabular}{ll}
\hline Grados de libertad: 55 & $t$ \\
\hline Autoconcepto & $-2.9469 \mathrm{e}-15$ \\
\hline Autorrealización & $-1.1188 \mathrm{e}-14$ \\
\hline Autoconciencia emocional & $-1.5251 \mathrm{e}-14$ \\
\hline Expresión emocional & $7.5262 \mathrm{e}-15$ \\
\hline Asertividad & $3.7704 \mathrm{e}-15$ \\
\hline Independencia & $-5.0101 \mathrm{e}-15$ \\
\hline Relaciones interpersonales & $-1.8147 \mathrm{e}-14$ \\
\hline Empatía & $5.3677 \mathrm{e}-15$ \\
\hline Responsabilidad social & $-1.0058 \mathrm{e}-14$ \\
\hline Resolución de problemas & $-4.372 \mathrm{e}-15$ \\
\hline Prueba de la realidad & $-2.3215 \mathrm{e}-15$ \\
\hline Control de impulsos & $7.3555 \mathrm{e}-15$ \\
\hline Flexibilidad & $1.0615 \mathrm{e}-14$ \\
\hline Tolerancia al estrés & $2.0545 \mathrm{e}-15$ \\
\hline Optimismo & $1.7611 \mathrm{e}-13$ \\
\hline
\end{tabular}

Fuente: elaboración propia. 


\section{DISCUSIÓN}

El trabajo realizado se extiende transversalmente sobre los comportamientos equidistantes en el líder, esto debido a que la centralidad del este genera como resultado que el subordinado estribe del alcance, creatividad e innovación suya. Estas dependencias reflejan satisfacción laboral, compromiso afectivo, bienestar psicológico y motivación de los subordinados, consecuencias que se relacionan con los resultados del comportamiento organizacional a largo plazo (Higgs y Dulewicz, 2016).

La prueba T indica que no hay evidencia de diferencia significativa entre los valores estandarizados para cado uno de los constructos después de la intervención de coaching, lo que implica que no se tienen elementos para afirmar que el coaching tuvo algún impacto en la IE del grupo de líderes estudiado. Si bien para cada uno de ellos se obtuvieron valores distintos y varios mostraron un mejor desempeño en su IE o en algunos de los constructos, esto no sucedió de manera homogénea para todos los líderes incluidos en el estudio, como se esperaba de acuerdo con lo que plantea la teoría respecto a la posibilidad de modificar las habilidades emocionales en los adultos (Petrides et al., 2016). Si bien existen estudios como el de Groves et al. (2008), que sostiene que existe evidencia empírica de que se puede desarrollar la IE mediante aplicaciones de coaching, al menos en el estudio realizado, bajo las condiciones específicas que se implementaron y para el grupo particular en el que se realizó, no se encontró soporte empírico suficiente para corroborarlo.

Es de subrayarse la importancia de comprender y manejar efectivamente las habilidades integradas en la IE para lograr ser un líder exitoso por las particulares relaciones con la autoeficacia, autodirección, correcto desempeño laboral y un exitoso e integral trabajo con el equipo (Mullen et al., 2018).

Koc (2019) postula que la IE es fundamental en los entornos turísticos por incrementar la eficiencia y los resultados del sector. En esto se incluyen las relaciones sociales positivas que son las encargadas de promover la generación de niveles altos de calidad en el servicio, que se convierten en elementos que pueden ser extremadamente notables en los colaboradores involucrados en el servicio para lograr mejores resultados con clientes externos e internos (Koc y Boz, 2020). Con esto se destaca que, a través de la IE, el ejercicio del liderazgo facilita generar y provocar una mayor satisfacción en la empresa turística de forma efectiva, consciente y comprometida.

El coaching es esencial en el desarrollo de la IE y la gestión de las relaciones interpersonales dentro de la organización hotelera y turística (Koc, 2019). Los resultados incluidos en este trabajo agregan valor a estas investigaciones al otorgar evidencia sobre la necesidad de construir intervenciones de coaching que, de acuerdo con Caruso y Rees (2019), son aún pocos los estudios realizados que sostengan esto. Aun así, el coaching se considera como una práctica altamente efectiva para el desarrollo de la IE (Human Capital Institute, 2013) y estos resultados advierten la importancia de construir estas intervenciones desde conceptualizaciones profundas e integrales.

Los líderes asimilan, cuando forman parte de una organización, cómo sentirse acerca de lo que hacen y lo que aprenden; la IE en el trabajo se refleja a través de las prácticas y experiencias laborales diarias (Higgs y Dulewicz, 2016). Elevados índices de IE reflejarán comportamientos colaborativos al interior de la empresa y favorables para los resultados buscados. Tamizando la teoría destacada en el estudio, se permite identificar como la IE favorece el ejercicio del liderazgo de la empresa turística, que desde 
los postulados por Reuven Bar-on (2006; 2013) se exterioriza de la siguiente forma: el Autoconcepto permite al líder percibirse, comprenderse y aceptarse a sí mismo; esto representa una postura de seguridad ante sus subordinados, quienes en consecuencia se sienten seguros y respaldados. Asimismo, la Autoconciencia emocional equipa al líder con la capacidad para comprender las propias emociones; esta habilidad, sumada a la Asertividad y a la Expresión emocional, le facilita el poder expresar de forma eficaz y constructiva las propias emociones, lo que genera en los subordinados contextos colaborativos desde la comprensión emocional y la creación de círculos productivos.

Por su parte, la Independencia permite al líder ser autosuficiente y libre de dependencia emocional de los demás para tomar decisiones y elegir los objetivos a seguir, habilidad relevante para entornos de colaboración expuestos a ritmos acelerados y necesitados de una capacidad de respuesta pronta y expedita como lo es la empresa turística. Esta, sumada a la Resolución de problemas, facilita el resolver eficazmente problemas de carácter personal e interpersonal. Es destacable la Prueba de la realidad para todos los contextos de decisión del líder, pues le permite validar objetivamente los sentimientos y pensamientos propios y poder diferenciarlos con la realidad exterior.

Los entornos laborales, en este contexto los referentes de la empresa turística, dependen de que el líder cuente con una alta Tolerancia al estrés para poder gestionar las emociones de forma eficaz y constructiva y disminuir o erradicar la posibilidad de sufrir un asalto emocional y explotar en consecuencia. También el Control de impulsos se abona a esta causa, permitiendo manejar las emociones propias de forma eficaz y constructiva.

Igualmente, referirse a la Flexibilidad es aludir a la habilidad para adaptar y ajustar los sentimientos y pensamientos del líder a nuevas situaciones. En la empresa turística es frecuente que las exigencias operativas requieran de nuevas y diferentes formas para responder a las necesidades y exigencias de los clientes, ahí es donde la flexibilidad marca una diferencia importante.

Por otro lado, la Empatía representa una habilidad imperdible para la compresión de los sentimientos propios y de los demás; esta favorece en el líder la comunicación proactiva y la cohesión de los equipos de trabajo hacia el logro de los objetivos, comprendiendo asertivamente las necesidades particulares de sus subordinados. Cuando la Empatía se enlaza con la Responsabilidad social, permite al líder identificarse y cooperar con el grupo, es consciente de los compromisos añadidos a su gestión y funciona en beneficio de los demás. Las Relaciones interpersonales favorecen al líder el poder establecer relaciones mutuamente satisfactorias y relacionarse bien con los demás, herramienta valiosa para establecer colaboraciones efectivas con sus subordinados, pares y directivos, además de fortalecer su capacidad de atención y servicio con sus clientes externos.

Adicionalmente, el Optimismo permite al líder ser positivo y mirar el lado bueno de la vida, favorece la proactividad en la empresa turística y a su vez se siente contento consigo mismo y con los demás. El líder requiere además de la Autorrealización, pues es precisamente la que le permite esforzarse para lograr las metas personales y poseer el deseo de actualizar su potencial y sus habilidades constantemente, elementos fructíferos para las empresas turísticas expuestas a la voraz competencia y necesidad de permanecer vigentes en el mercado.

Las emociones surgen durante las interacciones sociales, funcionan como un nódulo invisible que delimita los vínculos, por lo que se respalda que las habilidades integradas en la IE son significativas 
para el ejercicio del liderazgo en la empresa turística, pues generan mejores vínculos productivos e impactan positivamente la calidad de los servicios del turismo (Salisu et al., 2019).

\section{CONCLUSIONES}

En concordancia con diversas teorías consultadas y citadas en este trabajo, la relación entre IE y liderazgo es estrecha e implica los resultados y el éxito de la empresa; en este caso específico, de la empresa turística. Sin embargo, el propósito del estudio sobre determinar si existía una influencia significativa en la IE de un grupo de líderes de la empresa turística después de una intervención de coaching enfocado en estas habilidades, no se sostiene de acuerdo con los resultados obtenidos. Potencializar las habilidades emocionales de los lideres es tarea ardua y delicada que requiere de estructuras profundas. Una intervención de coaching grupal de doce horas, o tal vez menos, con base en estos resultados, no refleja una mejora significativa, por lo que sigue siendo necesario afinar las características del coaching mismo, las horas de impartición y la integración de los grupos de participantes, con lo que se concluye que no se logró una influencia positiva en la IE del grupo de líderes estudiado.

Este dato podría ser relevante para los procesos de coaching de las empresas de consultoría y a su vez, sus principales clientes, la empresa turística de la Riviera Maya, pues éstas prefieren normalmente intervenciones cortas, o de máximo ocho horas. Las medidas de IE anuncian mejorar el rendimiento laboral si están desarrolladas. Cabría considerar la posibilidad que intervenciones de poca duración pasen directamente a ser un gasto para la empresa turística y no una inversión.

La herramienta Reuven Bar-on muestra componentes con aceptable homogeneidad y consistencia, lo que le confieren a la escala altos valores de fiabilidad y validez, lo cual indica que resulta conveniente seguir utilizando esta herramienta, independientemente de que se trate de grupos pequeños de líderes.

Este trabajo contribuye con la literatura ya existente conceptualizando las influencias de la IE en el ejercicio del liderazgo en la empresa turística, buscando tamizar su comprensión desde un punto sintetizado. Cabe destacar que esta propuesta es una primera aproximación, pero es posible prefigurar la trascendencia de reflexionar sobre ella con base en sus supuestos, generar nuevas líneas de investigación para que los argumentos de éxito en la empresa turística logren viabilidad y determinar beneficios, considerando toda la diversidad para estructurar sociedades dentro de márgenes sostenibles.

Será necesario, en futuros estudios similares al que aquí se presenta, considerar diversas posibilidades de una intervención de coaching para lograr influencias significativas en la IE de los grupos de líderes, tales como la homogeneidad del grupo elegido, la duración de la intervención, el diseño instruccional de la intervención, así como situaciones contextuales que pudieran o no intervenir en la influencia analizada.

Asimismo, conviene considerar como línea futura una investigación donde se incluya determinar la IE y la felicidad de los líderes como variables relevantes para el desempeño, el logro de objetivos y el bienestar, integrando modelos de coaching diferentes para medir el impacto con base en su 
metodología, y conjuntamente complementar la investigación cuantitativa con investigación cualitativa para mejorar la autenticidad de los resultados de la investigación y proporcionar descripciones más ricas de los procesos y resultados de la intervención de coaching.

\section{AGRADECIMIENTOS}

Al Consejo Nacional de Ciencia y Tecnología (Conacyt) de México por su apoyo financiero para la realización de la investigación.

\section{CONFLICTOS DE INTERÉS}

Los autores declaran que no presentan conflictos de interés financiero, profesional o personal que pueda influir de forma inapropiada en los resultados obtenidos o las interpretaciones propuestas.

\section{CONTRIBUCIÓN DE AUTORES}

Para el desarrollo de este proyecto todos los autores han realizado una contribución significativa especificada a continuación:

César Omar Velázquez-Vega: idea, diseño del estudio, modelo estadístico y recogida de datos, además de la redacción del borrador del artículo.

Alfonso González-Damián: análisis e interpretación de los datos, la crítica de su contenido intelectual sustancial y la revisión detallada de los resultados estadísticos aplicados.

\section{REFERENCIAS}

Abravanel, M., Gavin, J. (2017). Exploring the evolution of coaching through the lens of innovation. International Journal of Evidence Based Coaching and Mentoring, v. 15, n. 1, 24-41. URL

Acosta Palomeque, G. R., Aviles Léon, B. E., Torres Fernández, J. P. (2017). Gestión Emocional: Factor Crítico de la Competitividad Emocional en el Profesorado Universitario. INNOVA Research Journal, v. 2, n. 10, 132-146. https://doi.org/10.33890/innova.v2.n10.2017.490

Ahmad, S., Nisar, Q., Othman, N., Mustafa Kamil, B. (2017). Do emotional intelligence \& Organizational politics influence the employee work behaviors and attitudes? Mediating role of political skill. Journal Pengurusan, v. 51, 1-16. $\underline{\text { URL }}$

Ariza, M. (2016). Tonalidades emocionales en la experiencia de la migración laboral Humillación y degradación social. En Ariza, M. Emociones, afectos y sociología. Diálogos desde la investigación social y la interdisciplina (279-325). Universidad Nacional Autónoma de México. 
Ashkanasy, N. M., Humphrey, R. H. (2011). Current emotion research in organizational behavior. Emotion Review, v. 3, n. 2, 214-224. https://doi.org/10.1177/1754073910391684

Ashkanasy, N. M., Humphrey, R. H. (2014). Leadership and Emotion: A Multilevel Perspective. En David V. Day (Ed.), The Oxford Handbook of Leadership and Organizations (pp. 1-39). Oxford University Press. https://doi.org/10.1093/oxfordhb/9780199755615.013.038

Athanasopoulou, A., Dopson, S. (2018). A systematic review of executive coaching outcomes: Is it the journey or the destination that matters the most? The Leadership Quarterly, v. 29, n. 1, 70-88. https://doi.org/10.1016/j.leaqua.2017.11.004

Băeşu, C., Bejinaru, R. (2015). Innovative Leadership Styles and the Influence of Emotional Intelligence. The USV Annals of Economics \& Public Administration, v. 15, n. 3, 136-145. URL

Baquero Barato, J. A., Rodríguez-Moneo, M. (2016). La relación entre el proceso de autorregulación y el proceso de coaching. Universitas Psychologica, v. 15, n. 1, 141-52. https://doi.org/10.11144/Javeriana.upsy15-1.rpap

Bar-On, R. (2006). The Bar-On model of emotional-social intelligence (ESI). Psicothema, n. 18, 13-25. $\underline{U R L}$

Bar-On, R. (2013). The 15 factors of the Bar-On model. URL

Bauman, D. C. (2018). Plato on Virtuous Leadership: An Ancient Model for Modern Business. Business Ethics Quarterly, v. 28, n. 3, 251-274. https://doi.org/10.1017/beq.2017.31

Bermúdez-Tirado, S., Ramírez-Hoyos, M. A., Jaramillo-Arias, A. (2016). Preferencias en el uso del tiempo libre de los estudiantes universitarios con enfoque en el sector turismo. Revista CEA, v. 2, n. 4, 75-88. https://doi.org/10.22430/24223182.173

Bernarás, E., Garaigordobil, M., de las Cuevas, C. (2011). Inteligencia emocional y rasgos de personalidad: Influencia de la edad y el género durante la edad adulta y la vejez. Boletín de Psicología, n. 103, 75-88. URL

Bisquerra Alzina, R., Martínez Olmo, F., Obiols Soler, M., Pérez Escoda, N. (2006). Evaluación de 360o: Una aplicación a la educación emocional. Revista de Investigación Educativa, v. 24, n. 1, 187203. URL

Bonesso, S., Gerli, F., Pizzi, C. (2015). The interplay between experiential and traditional learning for competency development. Frontiers in Psychology, v. 6, 1305. https://doi.org/10.3389/fpsyg.2015.01305

Boyatzis, R. E., Thiel, K., Rochford, K., Black, A. (2017). Emotional and social intelligence competencies of incident team commanders fighting wildfires. The Journal of Applied Behavioral Science, v. 53, n. 4, 498-516. https://doi.org/10.1177/0021886317731575 
Carlson, J., Geisinger, K., Jonson, J. (2014). The Nineteenth Mental Measurements Yearbook. Buros Mental Measurements Yearbook Series.

Caruso, D., Rees, L. (2019). Developing Leaders of Character with Emotional Intelligence. The Journal of Character \& Leadership Development, v. 6, n. 1, 43-51. URL

Chang, E. C. (2017). Applying the broaden-and-build model of positive emotions to social problem solving: Does feeling good (vs. feeling bad) influence problem orientation, problem-solving skills, or both? Journal of Social and Clinical Psychology, v. 36, n. 5, 380-395. https://doi.org/10.1521/jscp.2017.36.5.380

Cohen, E., Cohen, S. A. (2012). Current sociological theories and issues in tourism. Annals of Tourism Research, v. 39, n. 4, 2177-2202. https://doi.org/10.1016/i.annals.2012.07.009

Corti, L., Gelati, C. (2020). Mindfulness and Coaching to Improve Learning Abilities in University Students: A Pilot Study. International journal of environmental research and public health, v. 17, n. 6, 1935. https://doi.org/10.3390/ijerph17061935

Cosans, C. E., Reina, C. S. (2017). The Leadership Ethics of Machiavelli's Prince. Business Ethics Quarterly, v. 28, n. 3, 275-300. https://doi.org/10.1017/beq.2017.13

Davis, S. K., Humphrey, N. (2012). Emotional intelligence predicts adolescent mental health beyond personality and cognitive ability. Personality and Individual Differences, v. 52, n. 2, 144-149. https://doi.org/10.1016/j.paid.2011.09.016

Dawda, D., Hart, S. D. (2000). Assessing emotional intelligence: Reliability and validity of the Bar-On Emotional Quotient Inventory (EQ-i) in university students. Personality and Individual Differences, v. 28, n. 4, 797-812. https://doi.org/10.1016/S0191-8869(99)00139-7

Diez Farhat, S., Sánchez, C. E. (2017). Influencia de la personalidad y la inteligencia emocional en los estilos de liderazgo. Revista Empresarial, v. 11, n. 43, 22-28. URL

DiPerna, J. C., Sandilos, L. E. (2014). Review of the Emotional Quotient Inventory 2.0. En J. Carlson, K. Geisinger, J. Jonson (Eds.), The nineteenth mental measurements yearbook (pp. 17-19). Buros Center for Testing.

Drucker, P. (2007). The Change Leader. National Productivity Review, v. 19, n. 2, 13-20. https://doi.org/10.1002/npr.4040190203

Edú Valsania, S., Moriano, J. A., Molero, F. (2016). Authentic leadership and intrapreneurial behavior: cross-level analysis of the mediator effect of organizational identification and empowerment. International Entrepreneurship and Management Journal, v. 12, 131-152. https://doi.org/10.1007/s11365-014-0333-4

Fayol, H. (1949). Administration Industrielle Et Générale. En General and Industrial Management. Sir Isaac Pitman \& Sons. 
Fernández Moya, M. (2016). Inteligencia emocional de la sustentabilidad. Ingeniería, v. 26, n. 1, 7989. $\underline{U R L}$

Fernández-Abascal, E. (2009). Emociones positivas, psicología positiva y bienestar. Facultad de Psicología, UNED.

Fragoso-Luzuriaga, R. (2015). Inteligencia emocional y competencias emocionales en educación superior, ¿̇un mismo concepto? Revista Iberoamericana de Educación Superior, v. 6, n. 16, 110125. https://doi.org/10.1016/i.rides.2015.02.001

Garcia Reinoso, N., Carreño Mendoza, Á. L., Doumet Chilan, N. Y. (2020). La Validación del Modelo de Gestión Sostenible para el Desarrollo Turístico en Vinculación Universidad-Comunidades Manabitas. Ecuador. Revista Investigación y Negocios, v. 13, n. 21, 37-51. https://doi.org/10.38147/invneg.v13i21.82

Gelaidan, H. M., Al-Swidi, A., Mabkhot, H. A. (2016). Employee Readiness for Change in Public Higher Education Institutions: Examining the Joint Effect of Leadership Behavior and Emotional Intelligence. International Journal of Public Administration, v. 41, n. 2, 150-158. https://doi.org/10.1080/01900692.2016.1255962

Ghoudani, K. E., Pulido-Martos, M., Lopez-Zafra, E. (2017). Measuring emotional intelligence in Moroccan Arabic: the Wong and Law Emotional Intelligence Scale. International Journal of Social Psychology, v. 33, n. 1, 174-194. https://doi.org/10.1080/02134748.2017.1385243

Goleman, D. (1995). La inteligencia emocional. Porqué es más importante que el coeficiente intelectual. Bantam Books.

González-Damián, A. (2017). La relación entre empresa turística y desarrollo sostenible en las teorías administrativas y sociales. Una propuesta esquemática para su abordaje. Dimensiones Turísticas, v. 1, n. 1, 111-142. https://doi.org/10.47557/PIVJ1645

González-Damian, A. (2018). Construcción social de la experiencia turística. Una coproducción de turistas y anfitriones basada en hechos reales. Bubok Publishing.

Grandey, A. A., Gabriel, A. S. (2015). Emotional Labor at a Crossroads: Where Do We Go from Here? Annual Review of Organizational Psychology and Organizational Behavior, v. 2, n. 1, 323-349. https://doi.org/10.1146/annurev-orgpsych-032414-111400

Gregory, S. A., Wiles, M. P. (2018). Professional Development for All Seasons: Executive Coaching Accelerates Your Leadership. Armed Forces Comptrolle, 58-61. URL

Groves, K. S., Pat McEnrue, M., Shen, W. (2008). Developing and measuring the emotional intelligence of leaders. Journal of Management Development, v. 27, n. 2, 225-250. https://doi.org/10.1108/02621710810849353 
Herrero Garcí, P., Carbonero Martín, M. A., Flores Lucas, V., Martín Antón, L. J. (2019). Inteligencia emocional y liderazgo auténtico en los cargos públicos locales españoles. International Journal of Developmental and Educational Psychology, v. 4, n. 1, 21-28.

https://doi.org/10.17060/ijodaep.2019.n1.v4.1490

Hibbert, P., Beech, N., Siedlok, F. (2017). Leadership formation: Interpreting experience. Academy of Management Learning and Education, v. 16, n. 4, 603-622. https://doi.org/10.5465/amle.2015.0243

Higgs, M., Dulewicz, V. (2016). Leading with Emotional Intelligence: Effective Change Implementation in Today's Complex Context (1st ed.). Palgrave Macmillan.

Hollander, E. P. (1980). Leadership and Social Exchange Processes. En Gergen, K., Greenberg, M., Willis R. (Eds.), Social Exchange (p. 35). Springer. https://doi.org/10.1007/978-1-4613-3087-5 5

Human Capital Institute. (2013). Leadership and Emotional Intelligence: The Keys to Driving ROI and Organizational Performance. Human Capital Institute.

Ibarra Michel, J. P. (2014). Sustentabilidad y Competitividad de la Industria Hotelera en México. Journal of Intercultural Management, v. 6, n. 1, 47-66. https://doi.org/10.2478/joim-2014-0004

Issah, M. (2018). Change Leadership: The role of emotional intelligence. SAGE Open. https://doi.org/10.1177/2158244018800910

Jacka, J. M. (2018). How's your EQ? Emotional intelligence can help auditors build and maintain positive, productive relationships throughout the organization. Internal Auditor, v. 75, n. 1, 3642. $\underline{U R L}$

Jafari, J. (2005). El turismo como disciplina científica. Política y Sociedad, v. 42, n. 1, 39-56. URL

Kim, H. J., Jeong, M. (2018). Research on hospitality and tourism education: Now and future. Tourism Management Perspectives, v. 25, 119-122. https://doi.org/10.1016/i.tmp.2017.11.025

Koc, E. (2019). Emotional intelligence in tourism and hospitality. CABI.

Koc, E., Boz, H. (2020). Development of hospitality and tourism employees' emotional intelligence through developing their emotion recognition abilities. Journal of Hospitality Marketing and Management, v. 29, n. 2, 121-138. https://doi.org/10.1080/19368623.2019.1608885

Koontz, H. (2000). Revisión de la jungla de la teoría administrativa. Academy of Management Review, v. 199, 55-74.

Koontz, H., Weihrich, H., Cannice, M. (2012). Administración: Una perspectiva Global y Empresarial (14 ed.). McGraw-Hill. 
Kotzé, M., Venter, I. (2011). Differences in emotional intelligence between effective and ineffective leaders in the public sector: an empirical study. International Review of Administrative Sciences, v. 77, n. 2, 397-427. https://doi.org/10.1177/0020852311399857

Ladyshewsky, R. K. (2017). Peer coaching as a strategy to increase learning and development in organisational life - a perspective. International Journal of Evidence Based Coaching \& Mentoring, v. 15, n. 1, 4-10. URL

Lam, W., Lee, C., Taylor, M. S., Zhao, H. H. (2018). Does Proactive Personality Matter in Leadership Transitions? Effects of Proactive Personality on New Leader Identification and Responses To New Leaders and Their Change Agendas. Academy of Management Journal, v. 61, n. 1, 245-263. https://doi.org/10.5465/amj.2014.0503

Larsson, G., Sandahl, C., Söderhjelm, T., Sjövold, E., Zander, A. (2017). Leadership behavior changes following a theory-based leadership development intervention: A longitudinal study of subordinates' and leaders' evaluations. Scandinavian Journal of Psychology, v. 58, n. 1, 62-68. https://doi.org/10.1111/sjop.12337

Lee, Y. H, Chelladurai, P. (2017). Emotional intelligence, emotional labor, coach burnout, job satisfaction, and turnover intention in sport leadership. European Sport Management Quarterly, v. 18, n. 4, 393-412. https://doi.org/10.1080/16184742.2017.1406971

Lopez-Zafra, E., Pulido-Martos, M., Berrios-Martos, P., Augusto-Landa, J. M. (2017). Does transformational leadership predict group emotional intelligence in work settings? International Journal of Social Psychology, v. 32, n. 3, 513-538. https://doi.org/10.1080/02134748.2017.1352170

Losch, S., Traut-Mattausch, E., Mühlberger, M., Jonas, E. (2016). Comparing the effectiveness of individual coaching, self-coaching, and group training: How leadership makes the difference. Frontiers in Psychology, v. 7, 629. https://doi.org/10.3389/fpsyg.2016.00629

Madanchian, M., Hussein, N., Noordin, F., Taherdoost, H. (2017). Leadership Effectiveness Measurement and Its Effect on Organization Outcomes. Procedia Engineering, v. 181, 10431048. https://doi.org/10.1016/i.proeng.2017.02.505

Maqbool, R., Sudong, Y., Manzoor, N., Rashid, Y. (2017). The Impact of Emotional Intelligence, Project Managers' Competencies, and Transformational Leadership on Project Success: An Empirical Perspective. Project Management Journal, v. 48, n. 3, 58-75. https://doi.org/10.1177/875697281704800304

Mathew, M., Gupta, K. S. (2015). Transformational Leadership: Emotional Intelligence. SCMS Journal of Indian Management, v. 12, n. 2, 75. URL

Mayer, J. D., Caruso, D. R., Salovey, P. (2016). The Ability Model of Emotional Intelligence: Principles and Updates. Emotion Review, v. 8, n. 4, 290-300. https://doi.org/10.1177/1754073916639667 
Mestre Navas, J. M., Pérez, N., González de la Torre, G., Núñez Lozano, J. J., Guil Bozal, R. (2017). The development of emotional intelligence optimizing cognitive abilities through the compulsory education. Contextos Educativos: Revista de Educación, n. 20, 57-75. http://doi.org/10.18172/con.3023

Mfikwe, N. M. G., Pelser, T. G. (2017). The significance of emotional intelligence and leadership styles of senior leaders in the South African government. Journal of Contemporary Management Issues, v. 22, n. 2, 115-126. https://doi.org/https://doi.org/10.30924/mjcmi/2017.22.2.115

Montolio, C. G. (2014). Coaching aplicado a la hostelería y el turismo. Informació Psicológica, n. 107, 66-77. https://doi.org/10.14635/IPSIC.2014.107.6

Mullen, P. R., Gutierrez, D., Newhart, S. (2018). School Counselors' Emotional Intelligence and Its Relationship to Leadership. Professional School Counseling, 1-12. https://doi.org/10.1177/2156759X18772989

Muñoz Maya, C. M., Díaz Villamizar, O. L. (2014). El Coaching y la transformación organizacional: una oportunidad para las Empresas y los Coaches. Suma de Negocios, v. 5, n. 11, 62-69. https://doi.org/10.1016/S2215-910X(14)70020-5

Nass Kunstmann, K., Merino, J. M. (2008). Natural experiment as a new quasi-xperimental design in social and health-related sciences. Ciencia y Enfermería, v. 14, n. 2, 9-12. https://doi.org/10.4067/S0717-95532008000200002

Noguera, A. E. (2017). Consideraciones teóricas implícitas en la formación docente. Dialética, v. 13, n. 1, 160-190.

Ochalski, S. (2016). The Moderating Role of Emotional Intelligence on the Relationship Between Transformational Leadership and Work Engagement. International Leadership Journal, v. 8, n. 2, 68-87. URL

Özdemir, K., Özkul, A. S. (2016). The Relationship Between Emotional Intelligence Dimensions and Leadership Practices: A Study on White-Collar Employees in Defense Industry. Süleyman Demirel Üniversitesi Iktisadi ve Idari Bilimler Fakültesi Dergisi, v. 21, n. 4, 1449-1463. URL

Parr, A. D., Lanza, S. T., Bernthal, P. (2016). Personality profiles of effective leadership performance in assessment centers. Human Performance, v. 29, n. 2, 143-157. https://doi.org/10.1080/08959285.2016.1157596

Passmore, J. (Ed.). (2010). Excellence in Coaching - The industry guide (2nd ed.). Replika Press Pvt Ltd.

Petrides, K. V., Mikolajczak, M., Mavroveli, S., Sanchez-Ruiz, M. J., Furnham, A., Pérez-González, J.C. (2016). Developments in Trait Emotional Intelligence Research. Emotion Review, v. 8, n. 4, 335341. https://doi.org/10.1177/1754073916650493 
Pliopas, A. (2017). Drawing the triangle: How coaches manage ambiguities inherited in executive coaching. Brazilian Administration Review, v. 14, n. 4, 1-23. https://doi.org/10.1590/1807-7692bar2017170050

Preston, G., Moon, J., Simon, R., Allen, S., Kossi, E. (2015). The relevance of emotional intelligence in project leadership. Journal of Information Technology \& Economic Development, v. 6, n. 1, 1640.

Prieto Egido, M. (2017). La psicologización de la educación: implicaciones pedagógicas de la inteligencia emocional y la psicología positiva. Educación XXI, v. 21, n. 1, 303-320. https://doi.org/10.5944/educxx1.20200

Priyanka, J., Taranjeet, D. (2016). The influence of transformational leadership and emotional intelligence on the organizational commitment. Journal of Commerce and Management Thought, v. 7, n. 3, 586-598. https://doi.org/10.5958/0976-478X.2016.00033.1

R Core Team. (2021). The R Project for Statistical Computing. $\underline{\text { URL }}$

Ravier, L. (2009). Matriz de creatividad y urgencia para definir cuándo y porque contratar un coach organizacional. En Jornadas Internacionales Mentoring \& Coaching (258-272).

Rekalde, I., Landeta, J., Albizu, E., Fernandez-Ferrin, P. (2017). Is executive coaching more effective than other management training and development methods? Management Decision, v. 55, n. 10, 2149-2162. https://doi.org/https://doi.org/10.1108/MD-10-2016-0688

Ross, R. (2006). Pensamiento sistémico y mapas de proceso: Una combinación natural. En Senge, P. M. La quinta disciplina en la práctica (194-199). Granica.

Salisu, B., Yuping, W., Awang, S. R., Jusoh, A., Abdul Jabar, N. (2019). A two-step cluster model of the Emotional Intelligence and Leadership preferences of Four-Star Hotel Employees. Journal Kemanusiaan, v. 17, n. 2, 27-34. URL

Salomaa, R., Mäkelä, L. (2017). Coaching for career capital development: A study of expatriates' narratives. International Journal of Evidence Based Coaching and Mentoring, v. 15, n. 1, 114132. $\underline{U R L}$

Sánchez, G. (2013). PLS Path Modeling with R. Trowchez Editions. URL

Sancho, A., Buhalis, D., Gallego, J., Mata, J., Navarro, S., Osorio, E., Pedro, A., Ramos, S., Ruiz, P. (2008). Introducción al Turismo. Organización Mundial del Turismo.

Sandoval Lentisco, C., López Martínez, O. (2017). Educación, psicología y coaching: un entramado positivo. Educatio Siglo XXI, v. 35, n. 1, 145-164. https://doi.org/10.6018/i/286261 
Semuel, H., Siagian, H., Octavia, S. (2017). The Effect of Leadership and Innovation on Differentiation Strategy and Company Performance. Procedia - Social and Behavioral Sciences, v. 237, 11521159. https://doi.org/10.1016/i.sbspro.2017.02.171

Seymour, N., Jansen, S., Feng, L., Ayres, S., Austin, Z., Seabrook, J. A., Jones, P. M. (2017). Impact of emotional intelligence coaching on job satisfaction of pharmacists during organizational changes. Journal of Hospital Administration, v. 6, n. 4, 39-45. https://doi.org/10.5430/jha.v6n4p39

Smith, S. (2017). Using a blended style of coaching. International Journal of Evidence Based Coaching and Mentoring, v. 15, n. 1, 65-77. URL

Stelter, R. (2014). Narrative-Collaborative Theory and Practice. En A Guide to Third Generation Coaching. Springer Dordrecht Heidelberg. https://doi.org/10.1007/978-94-007-7186-4

Sykes, J. B. (1990). The concise Oxford dictionary of current English (seventh). Oxford University Press.

Taylor, F. (1968). Principios de la administración científica (10th ed.). Herrero Hermanos.

Ugarriza, N. (2001). La evaluación de la inteligencia emocional a través del inventario de Bar-On (ICE) en una muestra de Lima Metropolitana. Persona, n. 4, 129-160. URL

Vallejo Altamirano, D., Abarca Carrasco, R., Uquillas Granizo, G., Ramírez Garrido, R. (2017). Inteligencia emocional y el neuroliderazgo en las Empresas públicas. Observatorio de La Economía Latinoamericana, n. 235. URL

Whitmore, J. (2011). Coaching. El método para mejorar el rendimiento de las personas. Editorial Paidós. 